\title{
A Z-type PGeP pincer germylene ligand in a T-shaped palladium(0) complex
}

Javier A. Cabeza, ${ }^{* a}$ Pablo García-Álvarez, ${ }^{a}$ Carlos J. Laglera-Gándara ${ }^{a}$ and Enrique Pérez-Carreño ${ }^{\text {b }}$

\begin{abstract}
A dipyrromethane-based germylene decorated with two phosphane groups has been used to prepare an unusual T-shaped palladium( 0 ) containing a PGeP pincer germylene that acts as a Ztype ligand. This compound is a strong reducing reagent, as it has been easily oxidized to germyl-palladium(II) derivatives with a gold(I) complex, $\mathrm{HCl}$ and $\mathrm{Ph}_{2} \mathrm{~S}_{2}$ through processes that involve a formal addition of a bond of the oxidant across the Ge-Pd bond.
\end{abstract}

Germylenes decorated with two phosphane groups having a potential PGeP pincer-type topology ${ }^{1}$ are a new class of compounds that are expected to combine the easy tunability of the electronic and steric properties of germylenes ${ }^{2}$ and phosphanes ${ }^{3}$ with the interesting applicability that pincer-type ligands have already demonstrated in organometallic chemistry and catalysis. ${ }^{4}$ Although only a few germylenes of this type have been reported to date (compounds $\mathbf{A}-\mathbf{F}$ in Fig. 1), ${ }^{5-10}$ their yet scarce transition metal derivative chemistry ${ }^{5-9,11}$ has already afforded very interesting results, including rare T-shaped PGeP chloridogermyl complexes of gold(I $)^{7,8}$ and silver(I). ${ }^{8}$

However, the transition metal chemistry of PGeP germylenes $\mathbf{A}-\mathbf{D}$ is dominated by reactions that, involving the insertion of the germylene $G e$ atom into $M-X$ bonds $(M=$ metal; $X=$ metal, halogen), lead to complexes comprising PGeP germyl ligands that no longer maintain the original germylene character (Fig. 2).$^{5-8,11}$ In addition, it is also known that the NHCstabilized ( $\mathrm{NHC}=\mathrm{N}$-heterocyclic carbene) $\mathrm{PGeP}$ germylene $\mathbf{E}$ has allowed the synthesis of tetrahedral nickel complexes in which a PGeP germylene acts as a tripod (facial) ligand (Fig. 2). ${ }^{9}$ No transition metal derivative of germylene $\mathbf{F}$ has yet been reported. ${ }^{10}$ Therefore, metal complexes containing a genuine PGeP pincer germylene ligand, with coplanar $\mathrm{M}, \mathrm{Ge}$ and both $\mathrm{P}$ atoms, are currently unknown.

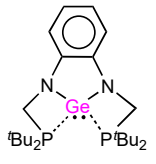

(A)

(Cabeza, 2017-2018)

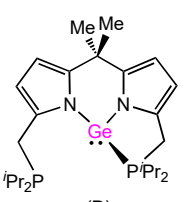

(D)

(Cabeza, 2019)

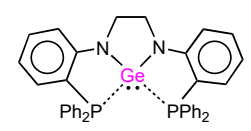

(B)

(Goicoechea, 2018)

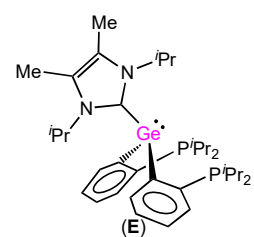

(Watanabe \& Tobita, 2019)

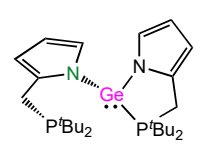

(C)

(Cabeza, 2019)

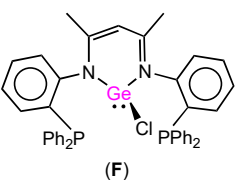

(Goicoechea, 2020)
Fig. 1 PGeP germylenes that are known to be precursors to PGeP pincer-type ligands.
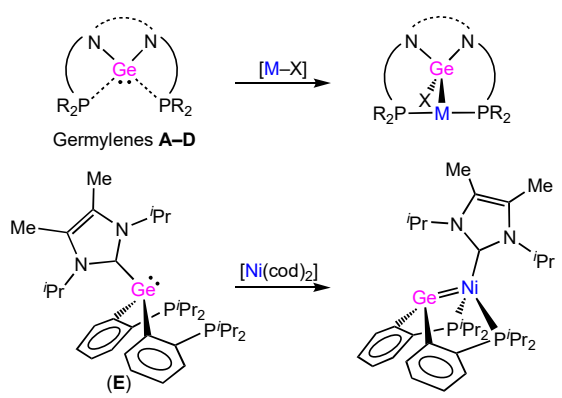

Fig. 2 Known reactivity of germylenes $\mathbf{A}-\mathbf{D}$ and $\mathbf{E}$ with transition metal complexes ( $\mathrm{X}=$ metal or halogen; $\mathrm{M}$ may be attached to additional ligands).

We now report the first metal complex supported by a PGeP pincer germylene. We also describe that this compound presents very unusual and interesting features: $(a)$ it is a rare Tshaped palladium( 0 ) complex, (b) its germylene fragment acts as a Z-type ligand and (c) it behaves as a very strong reducing reagent, as it can be easily oxidized to palladium(II) derivatives with mild oxidants, such as $[\mathrm{AuCl}(\mathrm{tht})]$ (tht = tetrahydrothiophene), $\mathrm{HCl}$ and $\mathrm{Ph}_{2} \mathrm{~S}_{2}$.

The diphosphane-germylene 5,5-dimethyl-1,9-bis(diisopropylphosphanylmethyl)dipyrromethane-N, $\mathrm{N}^{\prime}$ -

diylgermanium(II), Ge(pyrmPiPr ${ }_{2}{ }_{2} \mathrm{CMe}_{2}$ (D in Fig. 1$)^{8}$ reacted readily at room temperature with $\left[\mathrm{Pd}\left(\mathrm{PPh}_{3}\right)_{4}\right]$, displacing the four $\mathrm{PPh}_{3}$ ligands, to give $\left[\mathrm{Pd}\left\{\kappa^{3} P, G e, P-\mathrm{Ge}\left(\mathrm{pyrmP}^{i} \mathrm{Pr}_{2}\right)_{2} \mathrm{CMe}_{2}\right\}\right]$ (1), which was isolated as a very air- and moisture-sensitive dark green solid in $79 \%$ yield. The structure shown for this complex in Scheme 1 is based on its NMR spectra, DFT calculations and the results of subsequent reactivity studies.

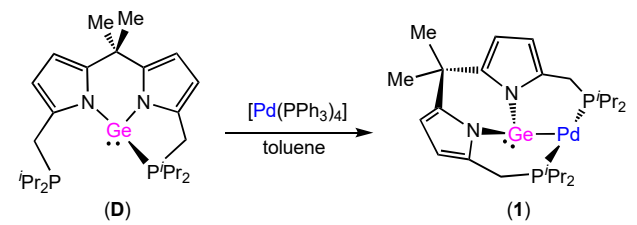

Scheme 1 Synthesis of complex 1.

The NMR spectra of complex 1 indicated the inequivalence of the $\mathrm{CMe}_{2}$ methyl groups, the existence of a symmetry plane and the absence of $\mathrm{PPh}_{3}$ ligands.

The T-shaped coordination environment of the palladium atom of complex 1 was indicated by a DFT study at the wB97XD/SDD/cc-pVDZ theory level (Fig. 3), which showed and almost lineal $\mathrm{P}-\mathrm{Pd}-\mathrm{P}$ arrangement $\left(175.35^{\circ}\right)$ and that the $\mathrm{Pd}-$ Ge distance $(2.507 \AA)$ is longer than the Pd-P distances (2.347 $\AA$ ) and also significantly longer than the $\mathrm{Pd}-\mathrm{Ge}$ distances found in non-pincer three-coordinate germylene-palladium(0) complexes, for example $\left[\mathrm{Pd}\left(\mathrm{PEt}_{3}\right)_{2}\left\{\mathrm{Ge}(\mathrm{HMDS})_{2}\right\}\right]$ (HMDS = $\mathrm{N}\left(\mathrm{SiMe}_{3}\right)_{2} ; \mathrm{Pd}-\mathrm{Ge} 2.333 \AA$ A $) .{ }^{12} \mathrm{~T}$-shaped palladium(0) complexes are very rare; in fact, we have found only three examples in the 
$\mathrm{CSD},{ }^{13}$ and they all involve a $\left[\mathrm{Pd}(\text { phosphane })_{2}\right]$ fragment attached to a very strong Lewis acid: $\left[\mathrm{Pd}\left(\mathrm{PBz}_{3}\right)_{2}\left(\mathrm{SO}_{2}\right)\right](\mathrm{Bz}=$ benzyl), ${ }^{14}\left[\mathrm{Pd}\left(\mathrm{PCy}_{3}\right)\left(\mathrm{I}^{t} \mathrm{Bu}\right)\left(\mathrm{AlCl}_{3}\right)\right]\left(\mathrm{Cy}=\right.$ cyclohexyl; $\mathrm{I}^{t} \mathrm{Bu}=1,3-$ bis(tertbutyl)imidazol-2-ylidene $)^{15} \quad$ and $\quad\left[P d\left\{\kappa^{3} P, B, P\right.\right.$ $\left.\left.\mathrm{BPh}\left(\mathrm{C}_{6} \mathrm{H}_{4} \mathrm{PC} y_{2}\right)_{2}\right\}\right],{ }^{16}$ the latter being a PBP pincer complex.

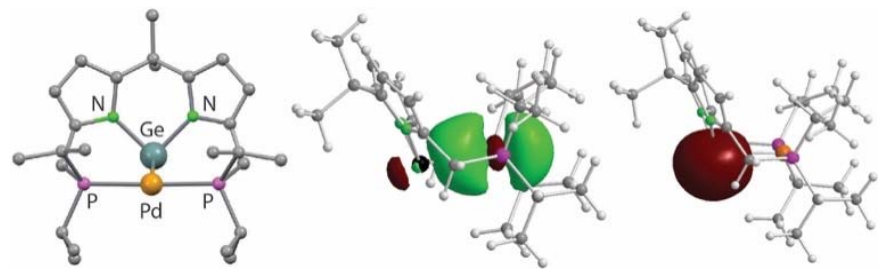

Fig. 3 DFT-optimized molecular structure (left; $\mathrm{H}$ atoms omitted for clarity) and NBO images of the HOMO (center) and HOMO-12-(right) orbitals of complex 1.

The unusual T-shaped coordination of the palladium atom and the fact that disubstituted (not donor-stabilized) germylenes present an ambiphilic character (they can behave as both Lewis acids and bases), ${ }^{17}$ led us to investigate the electronic structure of complex 1. A Natural Bond Orbital (NBO) analysis indicated that the HOMO of $\mathbf{1}$ (Fig. 3 ) is the molecular orbital responsible for the $\mathrm{Pd}-\mathrm{Ge}$ interaction, as it consists of a bonding interaction between a filled $d s$ hybrid orbital of the palladium and an empty $p$ orbital of the germanium (the latter is almost perpendicular to the $\mathrm{GeN}_{2}$ plane). The orbital holding the germylene lone pair, HOMO-12 (Fig. 3), is mostly constituted by a Ge $s$ orbital and does not participate in any bonding. Therefore, the germylene scaffold of complex 1 behaves as an uncommon $\sigma$-acceptor ligand $\left(Z\right.$-ligand $\left.{ }^{18}\right)$. This situation contrasts with that observed for the related nickel complex shown in Fig. 2 (derived from germylene E), which has been reported to present a $\mathrm{Ge}=\mathrm{Ni}$ double bond with a $\sigma$-donor $\pi$-acceptor behavior of the germylene fragment (bond length 2.2876(3) A). ${ }^{9}$ As far as we are aware, a Z-ligand behavior of a germylene has been previously claimed only once, for a diamidinatogermylene nickel(0) complex that serendipitously resulted in low yield from the reduction of a bis\{chloro(amidinato)germylene-phosphane\}nickel(0) complex with potassium metal, ${ }^{19}$ but the $\mathrm{Ni}-\mathrm{Ge}$ bond length of this complex, 2.2559(4) $\AA$, is unexpectedly much shorter than the $\mathrm{Pd}-\mathrm{Ge}$ bond length of complex 1 .

Complex 1 did not react with the strong $\sigma$-donor 4dimethylaminopyridine (dmap), confirming the unavailability of an accessible empty orbital on the Ge atom of 1 . Consequently, the reaction of $\left[\mathrm{Pd}\left(\mathrm{PPh}_{3}\right)_{4}\right]$ with the donor-stabilized germylene D.dmap, which was prepared on purpose (ESI), afforded a mixture of complex $1, \mathrm{dmap}$ and $\mathrm{PPh}_{3}$. This reactivity contrasts with the Lewis acid behavior observed for the nickel complex shown in Fig. 2 (derived from germylene $\mathrm{E}$ ), which is prone to add an NHC to its Ge atom. ${ }^{9}$

Attempting the displacement of tht from [AuCl(tht)] by the Ge lone pair of complex 1, these reagents were mixed in 1:2 mol ratio in THF. This reaction led to the precipitation of gold metal (violet solid) and to a solution that contained the new complex $\left[\mathrm{PdCl}\left\{\kappa^{3} P, G e, P-\mathrm{GeCl}\left(\mathrm{pyrmP}^{\mathrm{P}} \mathrm{Pr}_{2}\right)_{2} \mathrm{CMe}_{2}\right\}\right]$ (2; Scheme 2). The use of a $1: 11$ to $[\mathrm{AuCl}(\mathrm{tht})]$ mole ratio led to the incomplete consumption of 1 . Therefore, the reaction is a redox process in which the $\mathrm{Pd}^{0}$ atom of $\mathbf{1}$ is oxidized to $\mathrm{Pd}^{\prime \prime}$ by the $\mathrm{Au}^{\mathrm{l}}$ atom of [AuCl(tht)]. Alternatively, complex $\mathbf{2}$ was also prepared from germylene $\mathbf{D}$ and $\left[\mathrm{PdCl}_{2}(\mathrm{MeCN})_{2}\right]$ via an insertion of the Ge atom of $\mathbf{D}$ into a $\mathrm{Pd}-\mathrm{Cl}$ bond. An X-ray diffraction (XRD) structure (Scheme 2) confirmed that $\mathbf{2}$ is a square planar palladium(II) complex containing a PGeP pincer chloridogermyl ligand. ${ }^{7,11 c}$

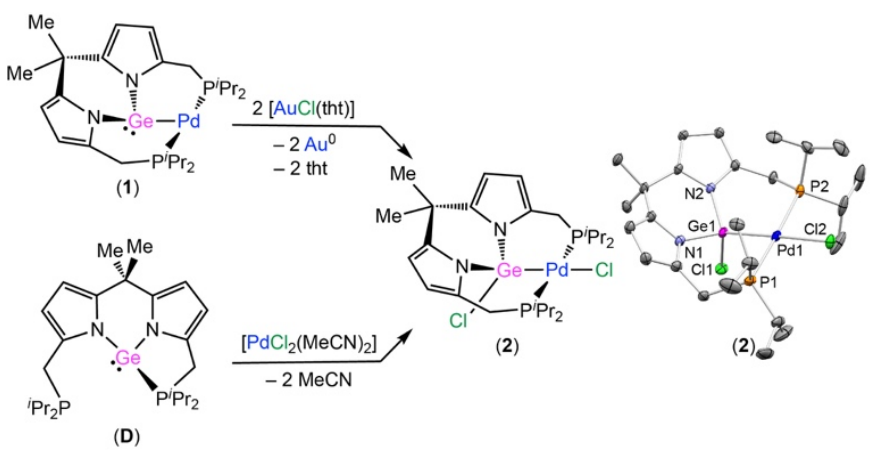

Scheme 2 Syntheses and XRD molecular structure of complex 2 ( $H$ atoms have been omitted for clarity; $20 \%$ displacement ellipsoids). Selected bond lengths ( $\AA$ ) and angles ( ${ }^{\circ}$ in 2: Pd1-Ge1 2.3001(4), Pd1-Cl2 2.3707(7), Pd1-P1 2.3138(9), Pd1-P2 2.3197(9), Ge1-Cl1 2.2070(8); Ge1-Pd1-Cl2 178.00(3), Pd1-Ge-Cl1 113.07(3).

As the formation of compound $\mathbf{2}$ from $\mathbf{1}$ and [AuCl(tht)] suggested a very strong reducing character for complex $\mathbf{1}$, we decided to investigate its use as a source of palladium(II) complexes by treating it with reagents that could oxidatively add to either the palladium atom (to end in germylenepalladium(II) complexes) or across the Ge-Pd bond (to end in germyl-palladium(II) derivatives). As a proof of concept, we report herein the reactions of complex 1 with hydrogen chloride and with diphenyl disulfide (Scheme 3). While the use of one equivalent of $\mathrm{HCl}$ (solution in diethyl ether) immediately led to the hydrido complex $\left[\mathrm{PdH}\left\{\kappa^{3} P, G e, P-G e(\mathrm{OH})\left(\mathrm{pyrmP}^{i} \mathrm{Pr}_{2}\right)_{2} \mathrm{CMe}_{2}\right\}\right]$ (3) Scheme 3), an excess of $\mathrm{HCl}$ led, as expected, ${ }^{20}$ to the chloride complex 2. The PdH signal of 3 was observed in its ${ }^{1} \mathrm{H}$ NMR spectrum as a triplet at $-5.09 \mathrm{ppm}$. The bis(phenylsulfide) complex $\left[\mathrm{Pd}(\mathrm{SPh})\left\{\kappa^{3} P, G e, P-G e(\mathrm{SPh})\left(\mathrm{pyrmP}^{i} \mathrm{Pr}_{2}\right)_{2} \mathrm{CMe}_{2}\right\}\right]$ Scheme 3), whose molecular structure was determined by XRD, was the only product of the reaction of 1 with $\mathrm{Ph}_{2} \mathrm{~S}_{2}$. These reactions confirm not only the strong reducing character of complex 1 but also that the oxidative addition of the appropriate oxidating reagent bond does not occur to the Pd atom (normal oxidative addition that would lead to germylenepalladium(II) products) but across the Ge-Pd bond, leading to germyl-palladium(II) derivatives. 


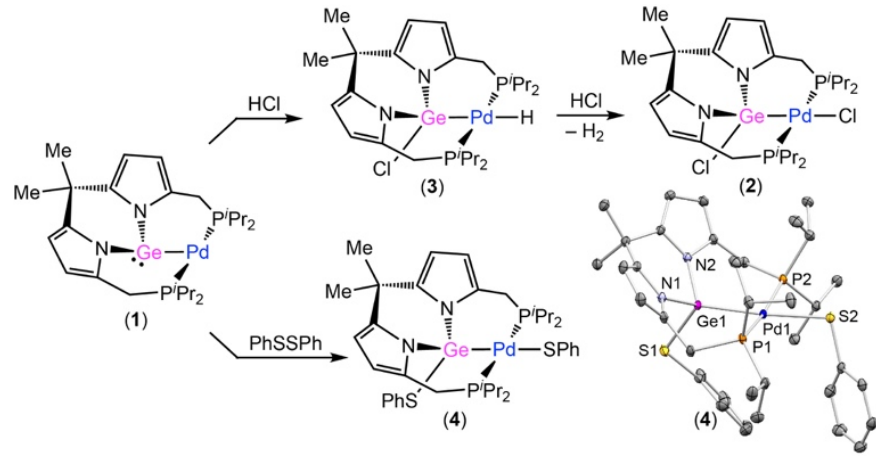

Scheme 3 Reactions of complex 1 with hydrogen chloride and diphenyl disulfide and XRD molecular structure of complex 4 (H atoms have been omitted for clarity; 50\% displacement ellipsoids). Selected bond lengths $(\AA)$ and angles $\left({ }^{\circ}\right)$ in 4: Pd1-Ge1 Pd1Ge1 2.3680(2), Pd1-S2 2.4003(4), Pd1-P1 2.3488(4), Pd1-P2 2.3343(4), Ge1-S1 2.2673(4); Ge1-Pd1-S2 175.35(1), Pd1-Ge-S1 121.94(1).

In conclusion, we have herein reported the synthesis of a palladium(0) compound (1) that is the first transition metal complex containing a genuine PGeP pincer germylene ligand, with coplanar $\mathrm{Pd}, \mathrm{Ge}$ and both $\mathrm{P}$ atoms. Interestingly, this complex (a) features an unusual T-shaped coordination geometry of its Pd atom, (b) contains a germylene moiety that behaves as a $\sigma$-acceptor Z-ligand, and (c) its reactivity is dominated by a very strong reducing character of its Ge-Pd scaffold, as 1 easily leads to germyl-palladium(II) complexes, upon reacting with $[\mathrm{AuCl}(\mathrm{tht})], \mathrm{HCl}$ and $\mathrm{Ph}_{2} \mathrm{~S}_{2}$, through processes that involve an uncommon addition of an oxidant bond across the Ge-Pd bond.

\section{Conflicts of interest}

There are no conflicts to declare.

\section{Acknowledgements}

This work was supported by MINECO (CTQ2016-75218-P, MAT2016-78155-C2-1-R and RED2018-102387-T) and AEI (PID2019-104652GB-I00) projects.

\section{References}

1 For a recent review on PGeP pincer complexes, see: J. A. Cabeza, P. García-Álvarez and C. J. Laglera-Gándara, Eur. J. Inorg. Chem., 2020, 784.

2 M. Asay, C. Jones and M. Driess, Chem. Rev., 2011, 111, 354.

3 (a) J. F. Hartwig, Organotransition Metal Chemistry: from Bonding to Catalysis, University Science Books, New York, 2010; (b) C. A. Tolman,
Chem. Rev., 1977, 77, 313; (c) Transition Metal Complexes of Phosphorus, Arsenic, and Antimony Ligands, ed. C. A. McAuliffe, J. Wiley, New York, 1973.

4 Selected recent reviews on pincer complexes and their applications: (a) Pincer Compounds: Chemistry and Applications, ed. D. MoralesMorales, Elsevier, Amsterdam, 2018. (b) E. Peris and R. H. Crabtree, Chem. Soc. Rev., 2018, 47, 1959; (c) The Privileged Pincer-Metal Platform: Coordination Chemistry \& Applications, ed. G. van Koten and R. A. Gossage, Springer, Cham, 2016; (d) M. Asay and D. MoralesMorales, Dalton Trans., 2015, 44, 17432; (e) C. Gunanathan and D. Milstein, Chem. Rev., 2014, 114, 12024; (f) The Chemistry of Pincer Compounds, ed. K. J. Szabó and O. F. Wendt, Wiley-VCH, Weinheim, 2014; (g) Organometallic Pincer Chemistry, ed. G. van Koten and D. Milstein, Springer, Heidelberg, 2013; $(h)$ G. Bauer and X. Hu, Inorg. Chem. Front., 2016, 3, 741; (i) H. A. Yonus, W. Su, N. Ahmad, S. Chen and F. Verpoort, Adv. Synth. Catal., 2015, 357, 283; (j) Catalysis by Pincer Complexes: Applications in Organic Synthesis and Catalysis, ed. K. J. Szabó and O. F. Wendt, Viley-VCH, Weinheim, 2014; (k) Q.-H. Dend, R. L. Melen and L. H. Gade, Acc. Chem. Res., 2014, 47, 3162; (I) The Chemistry of Pincer Compounds, ed. D. Morales-Morales and C. Jensen, Elsevier Science, Amsterdam, 2007.

5 L. Álvarez-Rodríguez, J. Brugos, J. A. Cabeza, P. García-Álvarez, E. Pérez-Carreño and D. Polo, Chem. Commun., 2017, 53, 893.

6 S. Bestgen, N. H. Rees and J. M. Goicoechea, Organometallics, 2018, 37, 4147.

7 J. A. Cabeza, I. Fernández, J. M. Fernández-Colinas, P. García-Álvarez and C. J. Laglera-Gándara, Chem. Eur. J., 2019, 25, 12423.

8 J. A. Cabeza, P. García-Álvarez, C. J. Laglera-Gándara and E. PérezCarreño, Dalton Trans., 2019, 48, 13273.

9 T. Watanabe, Y. Kasai and H. Tobita, Chem. Eur. J., 2019, 25, 13491.

10 S. Bestgen, M. Mehta, T. C. Johnstone, P. W. Roesky and J. M. Goicoechea, Chem. Eur. J., 2020, 26, 9024.

11 (a) J. Brugos, J. A. Cabeza, P. García-Álvarez, E. Pérez-Carreño and D. Polo, Dalton Trans., 2018, 47, 4534; (b) J. Brugos, J. A. Cabeza, P. García-Álvarez and E. Pérez-Carreño, Organometallics, 2018, 37, 1507; (c) L. Álvarez-Rodríguez, J. Brugos, J. A. Cabeza, P. GarcíaÁlvarez and E. Pérez-Carreño, Chem. Eur. J., 2017, 23, 15107.

12 Z. T. Cygan, J. E. Bender IV, K. E. Litz, J. W. Kampf and M. M. B. Holl, Organometallics, 2002, 21, 5373.

13 CSD version 5.41, updated August 2020

14 S. Arifhodzic-Radojevic, A. D. Burrows, N. Choi, M. McPartlin, D. M. P. Mingos, S. V. Tarlton and R. Vilar, J. Chem. Soc., Dalton Trans., 1999, 3981.

15 J. Bauer, H. Braunschweig, A. Damme, K. Gruss and K. Radacki, Chem. Commun., 2011, 47, 12783.

16 P. Steinhoff and M. E. Tauchert, Beilstein J. Org. Chem., 2016, 12, 1573.

17 (a) E. Rivard, Dalton Trans., 2014, 43, 8577; (b) L. Álvarez- Rodríguez, J. A. Cabeza, P. García-Álvarez and D. Polo, Coord. Chem. Rev., 2015, 300, 1.

18 A. Amgoune and D. Bourissou, Chem. Commun., 2011, 47, 859.

19 Z. Feng, Y. Jiang, H. Ruan, Y. Zhao, G. Tan, L. Zhang and X. Wang, Dalton Trans., 2019, 48, 14975.

20 The elimination of $\mathrm{H}_{2}$ has been observed upon protonation of a PCP pincer Pd"-H complex: R. Gerber, T. Fox and C. M. Frech, Chem. Eur. J., 2010, 16, 6771. 
Figure and Text for the Table of Contents

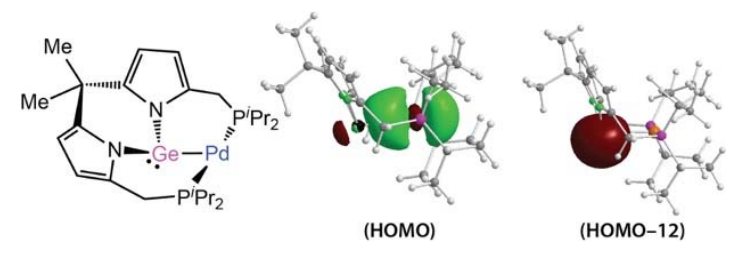

An unusual T-shaped palladium(0) complex having a $\sigma$-acceptor (Z-type) germylene scaffold has been prepared from a dipyrromethane-based PGeP germylene 